\title{
Análise da capacitação para o desenvolvimento de estudos Fase I pelos Centros de Bioequivalência certificados pela Anvisa no Brasil
}

\author{
Analysis of training for the development of Phase I studies by Anvisa certified \\ Bioequivalence Centers in Brazil
}

Luciana Neves de Carvalho ${ }^{1}$, Thomaz Augusto Alves da Rocha e Silva ${ }^{2}$, Luis Lopez Martinez ${ }^{3}$

\begin{abstract}
Resumo
O setor de Bioequivalência é fortemente regulamentado e consolidado no Brasil. Este fato é um ponto favorável na adequação dos Centros de Bioequivalência para a realização de Estudos Clínicos Fase I. Análise dos Centros de Bioequivalência nacionais certificados pela Anvisa, gerando informação sobre os pontos estratégicos, oportunidades e desafios para a realização de Estudos Clínicos Fase I no Brasil. Método: Estudo observacional, transversal, realizado através de um questionário eletrônico previamente aprovado pelo Comitê de Ética da Faculdade de Ciências Médicas da Santa Casa de São Paulo. O questionário foi enviado aos doze centros de Bioequivalência que conduzem estudos na Etapa Clínica e estavam certificados pela Anvisa em território brasileiro. O questionário foi aplicado através
\end{abstract}

1. Pós-Graduanda da Faculdade de Ciências Médicas da Santa Casa de São Paulo - Curso de Pós-Graduação Lato Sensu em Pesquisa Clínica e Medical Affairs

2. Professor da Faculdade Israelita de Ciências da Saúde Albert Einstein

3. Professor e Coordenador do Módulo de Medical Affair da Faculdade de Ciências Médicas da Santa Casa de São Paulo - Curso de Pós-Graduação Lato Sensu em Pesquisa Clínica e Medical Affairs e Gerente de Pesquisa Clínica no EPECLIN - Hospital das Clinicas, Faculdade de Medicina da Universidade de São Paulo - USP. (HCFMSP)

Trabalho realizado: Faculdade de Ciências Médicas da Santa Casa de São Paulo - Curso de Pós-Graduação Lato Sensu em Pesquisa Clínica e Medical Affairs

Endereço para correspondência: Luciana Neves de Carvalho. Faculdade de Ciências Médicas da Santa Casa de São Paulo - Curso de Pós-Graduação Lato Sensu em Pesquisa Clínica e Medical Affairs. Rua Dr. Cesário Mota Júnior, 61 - Vila Buarque -01221120 - São Paulo-SP - Brasil.E-mail: lunevescarvalho@gmail.com Conflito de Interesses, Financiamento E Aprovações Éticas: Declaramos que não temos nenhum conflito de interesse com o tema abordado, não contanto com nenhum financiamento e sob número de aprovação Ética CAAE 61922716.8.0000.5479, aprovado pelo Comitê de Ética (CEP) da Faculdade de Ciências Médicas da Santa Casa de São Paulo (FCMSCSP). do site SurveyMonkey ${ }^{\circledR}$ (www.surveymonkey.com) e foi estabelecido o prazo de quinze dias para resposta ao questionário. Resultados: Os entrevistados declararam-se como Pesquisadores Principais (40\%), Outros (40\%) e Coordenador do Centro (20\%). Todos os Centros de Bioequivalência participantes desta pesquisa foram criados há mais de cinco anos. Entre os entrevistados $80 \%$ declararam que a maior dificuldade do centro para a realização de estudos clínicos Fase I é a falta de demanda no Brasil e 20\% relatam que a maior dificuldade é a falta de investimentos dos próprios centros em Estudos Clínicos Fase I. Discussão: A falta de demanda do mercado nacional éo principal motivo apontado pelos Centros de Bioequivalência para a baixa realização de Estudos Clínicos Fase I no país, sendo que, todos os Centros de Bioequivalência participantes desta pesquisa demonstraram interesse em realizar esse tipo de Estudo Clínico. Conclusão: Ampliar seu portfólio de serviços requer investimentos de tempo, recursos fisicos e humanos, mas poderá representar uma solução viável tanto para os Centros de Bioequivalência, como para a realização de Estudos Clínicos Fase I. Futuros estudos serão necessários para ampliar e confirmar esta conclusão.

Descritores: Equivalência terapêutica, Agência Nacional de Vigilância Sanitária, Inovação, Brasil

\begin{abstract}
The Bioequivalence area is highly regulated and consolidated in Brazil. This is a favorable fact in the suitability of Bioequivalence Centers for Phase I Clinical Studies. Analysis of National Bioequivalence Centers certified by Anvisa, generating information on the strategic points, opportunities and challenges for conducting Phase I Clinical Studies in Brazil. Method: Cross-sectional observation study was performed through an electronic questionnaire approved by the Ethics Committee of Faculdade de Ciências Médicas da Santa Casa de São Paulo. The questionnaire was sent to twelve Bioequivalence Centers that conduct studies in the Clinical Phase which were certified by Anvisa in the Brazilian territory. The questionnaire was applied through the Survey Monkey ${ }^{\circledR}$
\end{abstract}


website (www.surveymonkey.com) and it was established a period of fifteen days to answer the questionnaire. Results: The interviewees declared themselves as Principal Investigator (40\%), Others (40\%) and Center Coordinator (20\%). All the Bioequivalence Centers participating in this research have been operating for more than five years. Among the interviewees $80 \%$ declared that, the greatest difficulty of the Center to conduct Phase I Clinical Studies is the lack of demand in Brazil and 20\% report that the greatest difficulty is the lack of investments of the centers themselves in Phase I Clinical Studies. Discussion: The lack of demand in the national market is the main reason pointed out by the Bioequivalence Centers for the low achievement of Phase I Clinical Trials in the country, and all the Bioequivalence Centers participating in this research have demonstrated interest in conducting this type of Clinical Study. Conclusion: The enhancement of services portfolio requires investments of time, physical and human resources, but it might represent a viable solution for the Bioequivalence Centers and for the accomplishment of Phase I Clinical Studies. Future studies will be needed to broaden and confirm this conclusion.

Keywords: Therapeutic equivalency, Brazilian Health Surveillance Agency, Innovation, Brazil

\section{Introdução}

A política de medicamentos genéricos (Lei $\mathrm{n}^{\circ}$ 9.787, 1999) foi realizada no Brasil, através da Lei dos Genéricos com o objetivo de estimular a concorrência comercial, melhorar a qualidade dos medicamentos e facilitar o acesso da população ao tratamento medicamentoso. Permitiu também a introdução e discussão de conceitos nunca antes utilizados para o registro de medicamentos no Brasil: Biodisponibilidade, Bioequivalência, Equivalência Farmacêutica, Medicamentos Genéricos, Sistema de Classificação Biofarmacêutica e Bioisenção ${ }^{(1)}$. A partir de então, diante dessa nova necessidade no cenário nacional, surgiu também uma nova oportunidade de mercado e de negócios: os Centros de Bioequivalência.

O primeiro registro de um Centro de Bioequivalência certificado pela Anvisa data de junho de 2001. Em 2016, dezesseis Centros de Bioequivalência estavam certificados no Brasil, sendo que somente doze destes centros realizam estudos na Etapa Clínica. Para atender a grande demanda e aumentar a oferta de serviços qualificados e certificados de Centros de Bioequivalência, a Anvisa igualmente certificou trinta e cinco centros de bioequivalência internacionais fora do Brasil para esta finalidade ${ }^{(2)}$.

Em 2018, até o presente momento, o país conta com quatorze centros de Bioequivalência habilitados pela Anvisa, sendo que somente onze deles conduzem estudos na Etapa Clínica ${ }^{(2)}$.
Conforme especificado na Resolução 24 de 14 de junho de 2011 publicada pela Anvisa, após o registro de um medicamento genérico e/ou similar, sua renovação deverá ser realizada somente a cada cinco anos. Assim, existe a possibilidade de que, após um volume inicial muito grande, ocorra a redução do número de estudos de bioequivalência/biodisponibilidade. Portanto, parte da infraestrutura física e de recursos humanos qualificados existentes para este setor, com o passar do tempo, poderá ficar ociosa ou subutilizada. De fato, entre os anos de 2011 e 2016, pelo menos quatro Centros de Bioequivalência que realizavam estudos na Etapa Clínica, foram fechados no Brasil(2).

A maior parte dos Estudos Clínicos realizados no Brasil são estudos Fase III e IV e grande parte dos Patrocinadores investe somente em Estudos de fase III. Essa é uma tendência nacional que corresponde à tendência mundial, em que apenas quando se adquire excelência em Estudos de Fase III é que o país começa a ser considerado para estudos mais complexos como Fase II e mesmo Fase I ${ }^{(3)}$.

Os aspectos éticos e regulatórios no Brasil são aderentes à legislação internacional, embora o prazo de aprovação seja, em média, superior aos demais países. Esse fator, em conjunto com a baixa capacidade de inovação da indústria brasileira e a incipiente infraestrutura para testes de fase I e II, constitui um dos principais desafios para o fortalecimento da pesquisa clínica no Brasil ${ }^{(4)}$.

A realização de Estudos Clínicos no Brasil é do interesse da sociedade, do governo, dos pacientes e de todos os setores envolvidos, sejam eles privados ou públicos, sendo estratégico para o sucesso da pesquisa e desenvolvimento (P\&D) de medicamentos inovadores ${ }^{(5)}$.

Com o recente movimento das empresas nacionais em direção a produtos inovadores, essa questão torna-se ainda mais relevante. $\mathrm{O}$ desejável movimento de mudança do perfil dos testes realizados no país, com ampliação dos testes de fase I e II, exigirá a capacitação para realização de ensaios em fases iniciais de empresas e profissionais brasileiros, principalmente no desenho de protocolos clínicos ${ }^{(4)}$.

Considerando o atual consenso entre governo, sistema regulador, pesquisadores e setor privado (patrocinadores e investidores) em colocar a Pesquisa Clínica como estratégica para as políticas de P\&D e inovação no país, estarão os Centros e Pesquisa brasileiros preparados para atender a nova demanda e capacitados para a condução de Estudos Clínicos mais precoces como os Estudos de Fase I?

Nosso estudo analisou os Centros de Bioequivalência nacionais certificados pela Anvisa gerando informação sobre os pontos estratégicos, oportunida- 
des e desafios para a realização de Estudos Clínicos Fase I no Brasil.

\section{Metodologia}

Este estudo observacional, transversal, foi realizado através de um questionário eletrônico, que assim como o Protocolo de Pesquisa, foi previamente aprovado pelo Comitê de Ética (CEP) da Faculdade de Ciências Médicas da Santa Casa de São Paulo (FCMSCSP) sob número de CAAE 61922716.8.0000.5479.

Os Centros de Bioequivalência foram convidados a participar voluntariamente desta pesquisa científica. A decisão de quem seria designado para responder o questionário eletrônico, bem como, o critério usado para esta decisão, ficou inteiramente a critério de cada Centro de Bioequivalência. O questionário contendo perguntas que analisaram as características tanto do entrevistado quanto do centro foram enviados aos doze centros de Bioequivalência que conduzem estudos na Etapa Clínica e estavam certificados pela Anvisa em território brasileiro ${ }^{(2)}$.

Os participantes de pesquisa só tiveram acesso ao conteúdo do questionário após fornecerem seu consentimento através do Termo de Consentimento Livre e Esclarecido (TCLE).

O questionário foi aplicado através do site Survey Monkey ${ }^{\circledR}$ (www.surveymonkey.com) e foi estabelecido o prazo de quinze dias para resposta ao questionário. Foi realizada uma análise descritiva dos dados obtidos no questionário e a resposta de cada pergunta foi analisada utilizando Estatística Inferencial com teste de hipóteses, utilizando-se o programa Excel ${ }^{\circledR}$.

\section{Resultados}

A amostra inicial foi considerada como os doze Centros de Bioequivalência convidados. Entre os sete participantes que acessaram a tela inicial contendo o TCLE, todos consentiram em participar desta pesquisa, o que corresponde a $58,3 \%$ da amostra inicial. No entanto, obtivemos a adesão de cinco participantes em completar o questionário após o TCLE, ou seja, $41,6 \%$ do total.

\section{Características do Entrevistado}

Entre os entrevistados, dois declararam-se como Pesquisadores Principais (40\%), dois declararam-se como Outros (não especificado) (40\%) e um declarou-se como Coordenador do Centro (20\%).

Acerca dos conhecimentos de bioética, três (60\%) participantes declararam que adquiriram conhecimento através de cursos profissionalizantes e dois entrevistados (40\%) através de leitura orientada. A data do último curso ou treinamento em bioética foi há mais de dois anos para dois entrevistados (40\%), há mais de três anos para um (20\%), há mais de cinco anos para um (20\%) e um entrevistado (20\%) declarou que nunca realizou curso ou treinamento em bioética.

Entre os entrevistados, quatro $(80 \%)$ declararam que a principal fonte de conhecimento em Pesquisa Clínica foi adquirida através de cursos profissionalizantes e um entrevistado (20\%) declarou que adquiriu conhecimento através de congressos, palestras e conferências.

\section{Características do Centro}

Todos os Centros de Bioequivalência participantes desta pesquisa $(100 \%)$ foram criados a mais de cinco anos.

Entre os entrevistados, dois (40\%) declaram possuir mais de vinte e cinco membros permanentes, um $(20 \%)$ declarou ter entre treze a dezoito membros permanentes, um (20\%) declarou ter entre sete a doze membros permanentes e um $(20 \%)$ declarou ter de um a seis membros permanentes.

Quando questionados sobre ter um membro efetivo com formação específica em farmacovigilância quatro ( $80 \%$ ) Centros de Bioequivalência entrevistados declararam possuir, sendo que, um (20\%) declarou que não possui um integrante com formação específica nesta área de atuação.

Todos os entrevistados (100\%) declararam que o centro em questão possui um membro efetivo com formação específica na área de farmacologia.

Entre os entrevistados quatro (80\%) declararam que seus respectivos Centros de Bioequivalência fazem parte de instituições privadas e um $(20 \%)$ declarou fazer parte de uma instituição pública.

Sobre o número de estudos realizados no ano de 2016, dois (40\%) declararam que foi maior que vinte e quatro estudos; um (20\%) informou que o número foi doze; um entrevistado (20\%) informou que foram seis; e um (20\%) informou que o número anual de estudos foi menor que quatro, conforme ilustrado na Figura 1.

Entre os entrevistados, três (60\%) declararam não ter experiência com o envio de amostras biológicas para o exterior e dois (40\%) informaram ter essa experiência.

O número de colaboradores do Centro de Bioequivalência envolvido com a etapa clínica variou entre os entrevistados: dois ( $40 \%$ ) declararam que é de cinco a dez colaboradores; um (20\%) declarou que esse número é de vinte um a trinta, um $(20 \%)$ declarou que de onze a vinte e um entrevistado $(20 \%)$ declarou que o número de colaboradores é menor de cinco, conforme ilustrado na Figura 2. 


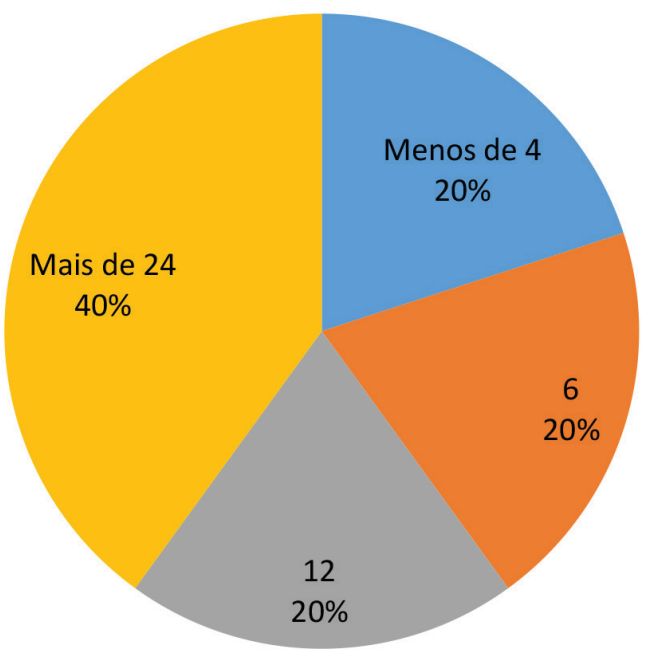

Figura 1 - Número de Estudos conduzidos em 2016.

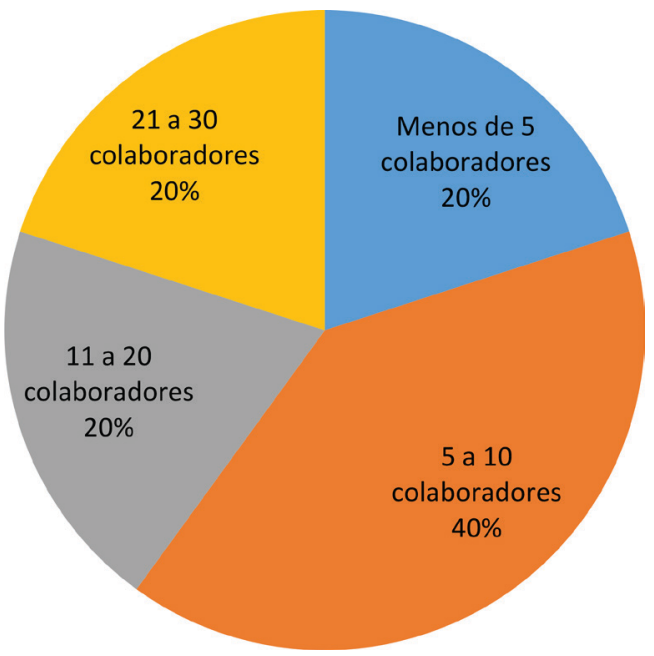

Figura 2 - Número de colaboradores envolvidos na Etapa Clínica.

Com relação ao número de colaboradores envolvidos com a etapa analítica, a maioria (três entrevistados totalizando $60 \%$ ) declarou ser de cinco a dez colaboradores, um (20\%) declarou que esse número é de vinte um a trinta e um entrevistado (20\%) declarou que esse número de colaboradores é menor de cinco.

Quanto ao número de analistas na equipe permanente, três entrevistados $(60 \%)$ responderam que seu centro possui mais de quatro analistas, um (20\%) informou que seu centro possui três analistas e um Centro de Bioequivalência (20\%) possui somente um analista na sua equipe permanente.

Entre os entrevistados, quatro $(80 \%)$ declararam que o Centro de Bioequivalência não está localizado dentro de um hospital e um centro entrevistado $(20 \%)$ declarou estar. Por outro lado, todos os centros entrevistados $(100 \%)$ responderam que possuem Unidade de Terapia Intensiva (UTI) disponível para atender emergências dos estudos, sendo que $75 \%$ (três centros) declaram ter contrato com UTI móvel para remoção dos voluntários, enquanto que $25 \%$ (um centro) respondeu negativamente a esta questão.

A média de tempo para aprovação dos Protocolos de Estudos de Bioequivalência pelo seu respectivo CEP é de até quinze dias em $40 \%$ dos Centros de Bioequivalência entrevistados, até trinta dias em 40 $\%$ dos centros e até quarenta e cinco dias em $20 \%$ dos centros participantes.

Entre os entrevistados, três (60\%) declararam já ter recebido inspeções de outras entidades e ou agências, porém não relacionadas ao Food and Drug Administration (FDA) ou European Medicines Agency (EMA), e dois entrevistados (40\%) declararam não ter recebido nenhuma outra inspeção além da Anvisa.

Quanto ao tema procedimento operacional padrão (POP) três $(60 \%)$ declararam que, a frequência de revisão de POP ocorre a cada dois anos, sendo que dois entrevistados $(40 \%)$ declararam que a frequência de revisão de POP é de três anos.

Entre os entrevistados, todos $(100 \%)$ declararam possuir mais de vinte leitos para condução dos estudos.

Os Centros de Bioequivalência foram questionados com relação à sua capacidade de operação, sendo que, um entrevistado (20\%) declarou que já opera no limite de sua capacidade máxima, um entrevistado (20\%) declarou que está atuando com apenas 10\% ou menos da capacidade, dois ( $40 \%$ ) declararam que estão atuando com apenas $25 \%$ ou menos da capacidade e um entrevistado (20\%) declarou que está atuando com apenas $50 \%$ ou menos da capacidade, conforme ilustrado na Figura 3.

Entre os entrevistados, três (60\%) declararam já ter conduzido estudos clínicos de Fase I e dois (40\%)

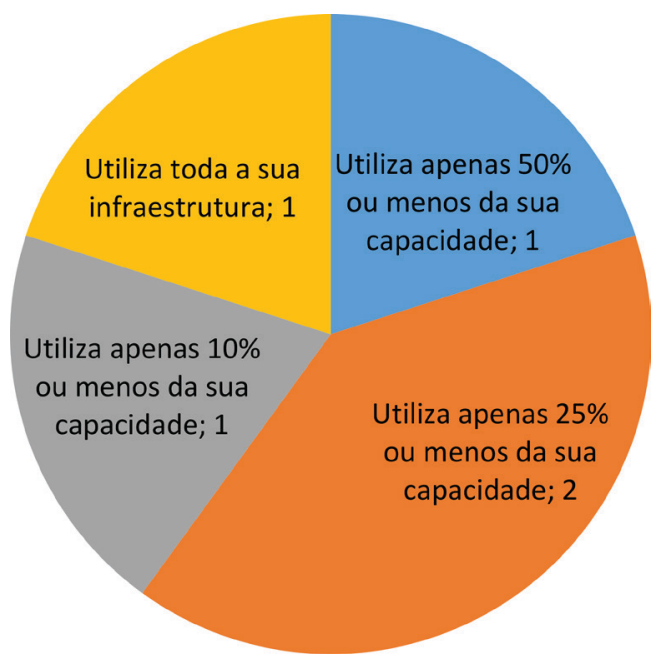

Figura 3 - Capacidade máxima dos centros utilizados na condução de estudos. 
declararam nunca ter conduzido esses estudos, conforme ilustrado na Figura 4.

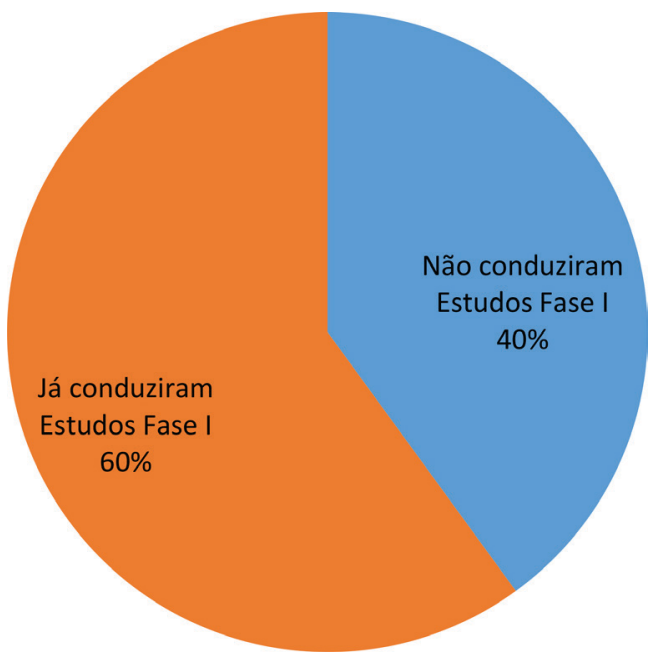

Figura 4-Centros que já conduziram Estudos de Fase 1.

Todos os entrevistados (100\%) declararam ter interesse em conduzir estudos clínicos Fase I e ter infraestrutura física adequada para a realização dos estudos clínicos Fase I. Ainda, quatro (80\%)declararam ter infraestrutura de recursos humanos para a realização destes estudos, enquanto um (20\%) declarou ter essa infraestrutura parcialmente.

Por fim, a maior dificuldade do centro para a realização de estudos clínicos Fase I, segundo quatro $(80 \%)$ dos entrevistados, é a falta de demanda no Brasil. Um entrevistado (20\%) declarou que a maior dificuldade é a falta de investimentos dos próprios centros em Estudos Clínicos Fase I, conforme ilustrado na Figura 5.

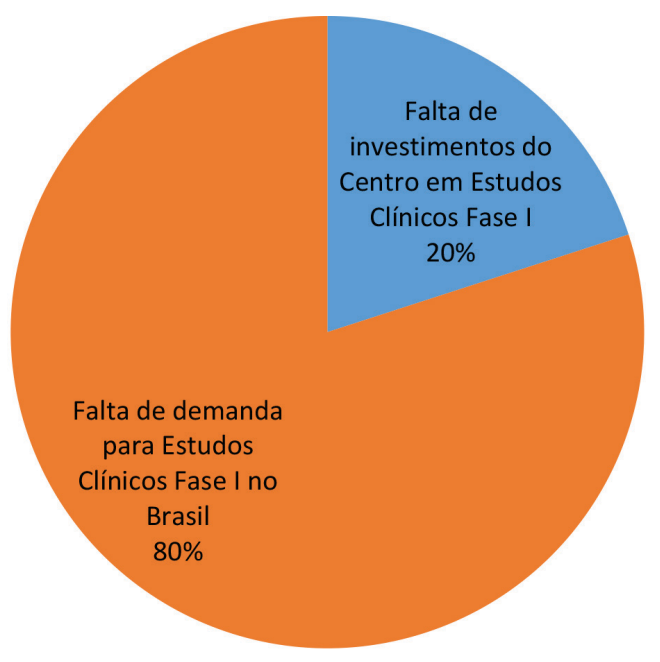

Figura 5 - Dificuldade para realização de estudos Fase I no Brasil.

\section{Discussão}

O recente reconhecimento da Anvisa pelo International Conference on Harmonization (ICH), ocorrida em novembro de 2016, confere credibilidade ao Brasil no cenário internacional da Pesquisa Clínica. O ICH reúne autoridades reguladoras e associações de indústrias farmacêuticas para discutir aspectos técnicos e científicos para o registro de medicamentos ${ }^{(6)}$.

\section{Representatividade e Relevância dos Resultados}

Esta pesquisa, sob nosso conhecimento, é um estudo pioneiro na análise dos Centros de Bioequivalência certificados que conduzem estudos na Etapa Clínica, aliando seu potencial para a realização de Estudos Clínicos Fase I no Brasil.

A maioria dos participantes desta pesquisa declarou atualmente exercer a função de Pesquisador Principal ou Coordenador do Centro de Bioequivalência entrevistado. Este fato reforça a validade das respostas encontradas nesta pesquisa, pois a maioria os Centros entrevistados foi representada por seu membro mais qualificado.

Da mesma forma, a maioria dos Centros de Bioequivalência participantes desta pesquisa declarou estar em funcionamento há mais de cinco anos. Este dado nos permite concluir que os Centros participantes desta pesquisa têm amplo conhecimento e experiência na área. Este fato agrega relevância às respostas obtidas por esta pesquisa.

Em nossa pesquisa tivemos um total de cinco participantes que responderam ao questionário. Apesar do interesse e da taxa de adesão dos convidados a participar desta pesquisa ter sido menor do que o esperado, foi possível a obtenção de uma amostra que representasse $41,6 \%$ do total de doze Centros de Bioequivalência que conduzem estudos na Etapa Clínica e estavam certificados pela Anvisa em território brasileiro.

\section{Capacitação dos Recursos Humanos dos Centros de Bioequivalência}

Para atuar em qualquer processo de investigação científica é imprescindível uma formação específica e de qualidade em Pesquisa Clínica e Boas Práticas Clínicas. Esses conhecimentos constituem um padrão de qualidade científica e ética internacional para o desenho, condução, registro e relato desses estudos ${ }^{(7)}$.

Segundo o Manual de Boas Práticas Clínicas, cada indivíduo envolvido na condução do estudo deve ser qualificado considerando-se educação, treinamento e experiência para a realização de suas respectivas $\operatorname{tarefas}^{(8)}$. 
Quanto ao tema conhecimento em Bioética os dados obtidos demonstram necessidade de maior atenção e investimentos na capacitação de recursos humanos envolvidos em pesquisa com seres humanos uma vez que $20 \%$ dos participantes desta pesquisa declararam nunca ter recebido treinamentos em Bioética. Além disso, $40 \%$ dos entrevistados declararam ter adquirido tais conhecimentos somente através de leitura orientada. Assim, os dados obtidos demonstram necessidade de maior atenção e investimentos na capacitação dos recursos humanos dos Centros de Bioequivalência.

Já no item conhecimentos em Pesquisa Clínica, a grande maioria dos participantes desta pesquisa (80\%) adquiriu tais conhecimentos através de cursos profissionalizantes, tendo realizado seu último treinamento entre um e cinco anos anteriores.

\section{Infraestrutura dos Centros de Bioequivalência}

A condução de um estudo de Bioequivalência requer o envolvimento de profissionais especializados nas áreas de farmacocinética, bioanálises e estatística, bem como, nas normativas que regulamentam a condução de Estudos Clínicos. Dessa forma, a multiplicidade de conhecimento promovida pela participação de especialistas de diversas áreas de atuação dentro do campo da saúde (farmacêuticos, médicos, enfermeiros, estatísticos, químicos, entre outros) no planejamento, execução e avaliação dos resultados, se apresenta como um parâmetro essencial para a adequada condução de um estudo de Bioequivalência ${ }^{(9)}$.

Com relação aos membros permanentes, $40 \%$ dos Centros de Bioequivalência participantes desta pesquisa possuem equipe permanente formada por mais de vinte e cinco membros, sendo que os outros $60 \%$ trabalharam com equipes reduzidas, o que pode ser resultado na baixa demanda no número de estudos de Bioequivalência.

\section{Produtividade e Capacidade de Operação dos Centros de Bioequivalência}

Um centro de pesquisa deve ser visto como uma empresa que presta um serviço e, como consequência, gera retorno financeiro. Quando pensamos em uma empresa, qualquer que seja o foco, temos de planejar estratégias que gerem recursos para sua manutenção e que ainda seja capaz de obter um lucro ${ }^{(10)}$.

De acordo com os dados disponíveis no site da Anvisa em dezembro de 2016, quatro Centros de Bioequivalência que realizavam Etapa Clínica e dez Centros de Bioequivalência que realizavam Etapa Analítica encerraram suas atividades nos últimos sete $\operatorname{anos}^{(2)}$.

Entre os Centros de Bioequivalência participantes desta pesquisa, há Centros que declaram realizar me- nos de quatro Estudos de Bioequivalência por ano. Isso equivale a média de um Estudo de Bioequivalência há cada três meses, podendo este fato causar dificuldades na manutenção do negócio. Estes dados sugerem que a redução da demanda por Estudos de Bioequivalência e a falta de diversificação dos serviços oferecidos pelo Centro pode ser a causa do fechamento de centros que oferecem exclusivamente Bioequivalência.

A maior demanda apontada pelos Centros de Bioequivalência participantes desta pesquisa foi de $50 \%$ da sua capacidade máxima. A baixa demanda no número de Estudos de Bioequivalência pode justificar a não terceirização de etapas, o número reduzido de médicos, farmacêuticos, coordenadores de centros e enfermeiros permanentes nas equipes e a pouca experiência no envio de amostras para o exterior. Por outro lado, a redução das equipes e da infraestrutura dos Centros de Bioequivalência pode dificultar o atendimento de estudos de maior complexidade.

\section{Aprovações éticas, Farmacovigilância e Inspeções Regulatórias nos Centros de Bioequivalência}

O tempo entre a aprovação do protocolo e o relatório final do ensaio clínico é estimado em cerca de 40 meses para os estudos de Fase II e III e em cerca de 32 meses para os ensaios clínicos de Fase $\mathrm{I}^{(11)}$.

O Sistema de Gestão da Qualidade dos Centros de Bioequivalência já é fortemente implantado, pois sua certificação depende de sua estruturação de acordo com todos os requisitos exigidos por manuais, POP, normas e resoluções aplicáveis. O setor de Bioequivalência é fortemente regulamentado e consolidado no Brasil. Este fato é um ponto favorável na adequação dos Centros de Bioequivalência para a realização de Estudos Clínicos Fase I.

Por outro lado, nenhum dos Centros de Bioequivalência entrevistado possui experiência com inspeções de agências regulatórias internacionais como FDA e EMA, possuindo somente experiência com inspeções da agência nacional (Anvisa) ou monitorias de patrocinadores que acompanham a condução de seus próprios estudos.

Assim sendo, nos parece claro que a baixa demanda por Estudos Clínico Fase I relatada pelos Centros de Bioequivalência brasileiros está diretamente relacionada a falta de certificações pelas agências regulatórias internacionais que garantam a validação e aceitação de seus resultados pelos mercados externos.

\section{Capacitação e adequação dos Centros de Bioequivalência para a realização de Estudos Clínicos Fase I}

É interessante observar que 80\% dos Centros de 
Bioequivalência participantes desta pesquisa declaram ter membros permanentes com formação específica em farmacovigilância. Em se tratando da adequação dos Centros de Bioequivalência para assumirem igualmente a condução de Estudos Clínicos Fase I a notificação de eventos adversos sérios e o registro de todos os eventos ocorridos é um requisito mandatório. Neste sentido, a maioria dos Centros de Bioequivalência apresenta recursos humanos capacitados e especializados em farmacovigilância.

Além disso, todos os Centros de Bioequivalência entrevistados declaram ter pelo menos um membro efetivo em sua equipe com formação em farmacologia. Esse requisito é fundamental para elaboração, condução e análise dos resultados de Protocolos de Estudos Clínicos Fase I, onde as informações de farmacocinética e farmacodinâmica serão testadas pela primeira vez em seres humanos.

Alguns Centros de Bioequivalência (60\% dos entrevistados) relataram que já realizaram Estudos Clínicos Fase I, demonstrando alguma experiência prévia.

Estima-se que cerca de um terço dos os ensaios clínicos patrocinados pela indústria farmacêutica e de biotecnologia seja terceirizado para entidades especializadas chamadas de Organizações Representativas de Pesquisa Clínica (CROs, sigla em inglês), em vez de serem conduzidos pelas próprias empresas farmacêuticas ou em colaborações diretas com centros acadêmicos de pesquisa ${ }^{(12)}$.

Com o evidente crescimento do P\&D e Inovação nas indústrias nacionais, alavancado pelos incentivos e condições favoráveis ao desenvolvimento de medicamentos inovadores, é evidente que a demanda por Estudos Clínicos Fase I tende a crescer no Brasil. No entanto, estes investimentos são cada vez mais elevados e de alto risco de insucesso.

O custo médio para realizar um ensaio clínico, desde a aprovação do protocolo até o relatório final, é de aproximadamente US $\$ 3,4$ milhões para os estudos de fase 1, US\$ 8,6 milhões para a fase 2 e US $\$ 21,4$ milhões para fase $3^{(13)}$. Portanto, mesmo para o setor industrial nacional, ao realizar este investimento não é viável ou estratégico atender somente o mercado brasileiro.

A falta de demanda do mercado nacional é o principal motivo apontado pelos Centros de Bioequivalência para a baixa realização de Estudos Clínicos Fase I no país, sendo que, todos os Centros de Bioequivalência participantes desta pesquisa demonstraram interesse em realizar esse tipo de Estudo Clínico.

\section{Conclusão}

Apesar da realização de Estudos Clínicos Fase I no Brasil ainda não ser muito comum, a maioria dos Centros de Bioequivalência entrevistados relataram já ter realizado este tipo de Estudo Clínico, demonstrando alguma experiência prévia.

A capacitação e adequação de Centros de Bioequivalência para a realização de Estudos de Bioequivalência e Estudos Clínicos Fase I, poderá ser uma solução estratégica para a escassa oferta de Centros de Pesquisa Clínica Fase I no Brasil. Isto requer a otimização e aproveitamento dos grandes investimentos em recursos financeiros, físicos e humanos já realizados na área de Bioequivalência.

Portanto, o atual estudo sugere que é possível a adequação dos Centros de Bioequivalência que realizam Etapa Clínica para atender a nova demanda de Estudos Clínicos Fase I no Brasil, e essa adequação pode ser um caminho para a sobrevivência e continuidade das operações dos próprios Centros de Bioequivalência. Futuros estudos serão necessários para ampliar e confirmar esta conclusão.

Diversificar e ampliar seu portfólio de serviços requer investimentos de tempo, recursos físicos e humanos, mas poderá representar uma solução viável tanto para os Centros de Bioequivalência, como para a realização de Estudos Clínicos Fase I, sendo essa mudança de paradigma estratégica para o P\&D e Inovação no setor farmacêutico no Brasil.

\section{Referências}

1. Araújo LU, Albuquerque KT, Kato KC, Silveira GS, Maciel NR, Spósito PA, et al. Medicamentos genéricos no Brasil: panorama histórico e legislação. Rev Panam Salud Publica. 2010; 28(6):48092.

2. Brasil. Agência Nacional de Vigilância Sanitária (ANVISA). Centros de bioequivalência e Biodisponibilidade. [online]. Disponível em: http://portal.anvisa.gov.br/centros-de-bioequivalencia-e-biodisponibilidade [2 jun 2018].

3. Lima, K S, Vidal BB, Drummond A, Wainstein, AJA. Análise crítica de qualidade dos centros de pesquisa de Belo Horizonte. Ciênc Cult. 2014; 66(1):34-7.

4. Gomes RP, Pimentel VP, Landim AB, Pieroni JP. Ensaios clínicos no Brasil: competitividade internacional e desafios. BNDES Setorial. 2012; 36:45-84.

5. Martinez LL. P \&D de medicamentos inovadores: a importância da farmacogenética e as características da população brasileira. [online]. São Paulo: BIOTEC AHG; 2014. Disponível em: http:/ / www.biotec-ahg.com.br/index.php/pt/acervo-de-materias/ assuntos-diversos /841-pad-medicamentos-capacitacao-de-rh [4 dez 2017]

6. Brasil. Agência Nacional de Vigilância Sanitária (ANVISA). Anvisa é novo membro do ICH. [online]. Disponível em: http:/ / portal.anvisa.gov.br/rss/-/asset_publisher/Zk4q6UQCj9Pn/ content/id/3080115. [4 dez 2017]

7. Conselho Federal de Medicina. A pesquisa clínica no Brasil é lenta por falta de profissionais capacitados. Brasília: CFM; 2014. Disponível em http://portal.cfm.org.br/index.php?option=com_content\&view=article\&id=24833:a-pesquisa-clinica-no-brasil-e-lenta-por-falta-de-profissionais-capacitados\&catid $=46$ [17 jan 2017]

8. International Council Harmonization. International Conference on Harmonisation of Technical Requirements for Registration of Pharmaceuticals for Human Use. ICH harmonised tripartite 
guideline. Guideline for good clinical practice E6(R1). Current Step 4 version dated 10 June 1996. [online]. Available from: https://www.ich.org/fileadmin/Public_Web_Site/ICH_Products/Guidelines/Efficacy/E6/E6_R1_Guideline.pdf [2017 Dec 4]

9. Vieira NR, Campos DR. Manual de bioequivalência. São Paulo: Dendrix; 2011. 208p. (Série Pesquisa Clínica).

10. Lousana G. Boas práticas clínicas nos centros de pesquisa. $2^{a}$. ed. Rio de Janeiro: Revinter; 2008. 260 p.

11. Martin L, Hutchens M, Hawkins C, Radnov A. How much do clinical trials cost? Nat Rev Drug Discov. 2017; 16(6):381-2.
12. Jeong S, Sohn M, Kim JH, Ko M, Seo HW, Song YK, et al. Current globalization of drug interventional clinical trials: characteristics and associated factors, 2011-2013. Trials. 2017; 18(1):288.

13. Sertkaya A, Wong HH, Jessup A, Beleche T. Key cost drivers of pharmaceutical clinical trials in the United States. Clin Trials. 2016; 13(2):117-26

Trabalho recebido: $02 / 04 / 2018$

Trabalho aprovado: 14/06/2018 